\title{
25 AÑOS DE LA REVISTA LATINOAMERICANA DE PSICOLOGIA
}

De modo particular e indudable, la Revista Latinoamericana de Psicologia (RLP) resulta ser la antítesis de una característica distintiva de los países en vías de desarrollo $\rightarrow$ en todo caso, marcha resuelta por dicho camino- pues, contrariamente a una muerte temprana, como las miles de personas (mayormente niños) que corren dicho destino en Latinoamérica, sigue con vida y, lo que es más importante, alimentada cada año por un creciente número de colaboradores (ojalá que también asi de lectores) que contribuyen a su robustez y cada vez mayor desarrollo y madurez (los artículos que contiene provienen de autores de todo el mundo, además de los mayoritariamente latinoamericanos).

¿Por qué este comentario dramático? La respuesta es muy sencilla: la historia de la prensa psicológica en América Latina, es la lectura de una quimera que se halla sometida a dificultades varias: además de la principal y tradicionalmente económica, se suma a ella la inexperiencia y/o agotamiento de sus propulsores -que de una forma u otra expresa una ausencia de objetivos precisos-y otras más que, como señala Ardila, han hecho que en la región "... (hayan) nacido y muerto muchas revistas de psicología [...] Casi siempre se trata de publicaciones respaldadas por una asociación de psicólogos o por una universidad. Lo normal es que tengan una vida efímera (de 2 a 3 años), que sean sumamente irregulares en su publicación y que representen un gasto gigantesco para la entidad que las publica. Además que no llegan muy lejos, más allá del grupo que las creó con gran optimismo y con muy poco realismo"'. Por supuesto que en el Perú, la actividad publicista -psiquiátrica y psicológica- no ha sido ajena a esta realidad, León y Mariátegui así lo han hecho notar ${ }^{2}$.

La RLP, en cierto modo, es la continuación de publicaciones pioneras en tierras sudamericanas. Representa la cristalización de aquellas preocu-

1. Ardila, R. (1988). Los veinte primeros años de la Revista Latinoamericana de Psicología, Revista Latinoamericana de Psicologia. 20(3); 401-406.

2. León, R. y Mariátegui, J. (1987). Una contribución al estudio de la historia de la psiquiatría y la psicología peruanas: El index de los Archivos Peruanos de Higiene Mental, Revista de NeuroPsiquiatría. 50(3); 135-140 
paciones por instalar vías efectivas y prolongadas de comunicación entre psicólogos y publicaciones que ante sí lo pretendieron, desde los Anales de Psicología (primera publicación de psicología en la región), editada por la Sociedad Argentina de Psicología -fundada en 1908 por Horacio Piñero (1869-1919) y José Ingenieros (1877-1925), entre otros- y que apareciera en tres volúmenes: en 1910, 1911 y 1914, seguida 21 años después por su connacional: los Anales del Instituto de Psicologia (de la Facultad de Filosofía y Letras de la Universidad de Buenos Aires), publicación que durante la segunda mitad de la década del 30 y los umbrales de la siguiente -fue editada en tres volúmenes: en 1935, 1938 y 1941 - fuera para la época, la única revista psicológica en Sudamérica y, además, caracterizada por una intención panamericanista pues, como ha observado León ${ }^{3}$, bajo la dirección de Enrique Mouchet (1886-1977) publicaron en ella varios colaboradores de otros varios países sudamericanos como; Bela Szekely (18921955), Emilio Mira y López (1896-1964), Italo Américo Foradori (19051968), Julio Endara (1899-1969), así como los peruanos Walter Blemenfeld (1882-1967), Honorio Delgado (1892-1969) y Mariano Iberico (18921974)

Desde aquellos lejanos años, han sido múltiples los esfuerzos editoriales en el sur, centro y el norte de América Latina ${ }^{4}$. Entre las más antiguas y aún vigentes revistas psicológicas, tenemos a los Arquivos Brasileiros de Psicologia (fundado por E. Mira y López en 1949, llamándose originalmente "Arquivos Brasileiros de Psicotecnia") y Acta Psiquiátrica y Psicológica de América Latina (fundada y aún dirigida por Guillermo Vidal desde 1954, habiéndose llamado inicialmente "Acta Neuropsiquiátrica Argenti$\left.n a^{3}\right)$ sumándose a ellas -y como ellas, andando también ya en relativa longevidad- la más importante y representativa revista de habla castellana: la Revista Latinoamericana de Psicologia .

3. León, R. (1988). Los "Anales del Instituto de Psicología” a 50 años de su fundación, Psicología y Sociedad. 1(1); 243-247

4. Una visión al respecto es revelada a través de la (dicho sea de paso, incompleta) Lista de revistas de psiquiatria, psicología y psicoanálisis de América Latina, países del Caribe y peninsula ibérica (1989) (Publicación No. 22 de la Fundación Acta, Fondo para la salud mental-Representación OPS/OMS- Argentina, 1990)

5. Otra revista internacional antecedente a la $R L P$ es el Interamerican Journal of Psychology/Revista Interamericana de Psicología, órgano de expresión de la Sociedad Interamericana de Pिsicologia, publicada desde 1967 y vigente hasta hoy, incluyendo artículos en castellano, portugués e inglés. 
Editada en la capital de Colombia (Santafé de Bogotá), la RLP contiene un Comité Editorial dentro del cual están representados los siguientes países: Argentina, Bolivia, Brasil, Colombia, Costa Rica, Cuba, Chile, Ecuador, El Salvador, Guatemala, México, Nicaragua, Panamá, Paraguay, Perú, Puerto Rico, República Dominicana, Trinidad y Tobago, Uruguay y Venezuela. Está compuesta de tres secciones: 1 . Artículos, que se publican en castellano y ocasionalmente en portugués según el estilo propuesto por la American Psychological Association (revisión de 1983). 2. Libros, que incluye reseñas de obras recientes y una sección sobre los libros recibidos y 3. El mundo de la psicología, con noticias de Congresos, actividades científicas, pequeñas biografias, necrología, etc. La $R L P$ publica aproximadamente, en promedio, 450 páginas anuales y circula por todo el mundo.

Una idea más clara de la activa continuidad de la $R L P$, puede ser observada en el cuadro de la página siguiente.

Ha transcurrido un cuarto de siglo desde que en Enero de 1969 apareciera el primer número de la $R L P$ y, desde entonces, no ha cesado de aparecer "puntualmente" tres veces al año registrando hasta fines de 1993, un total de 72 ejemplares publicados -19 de los cuales fueron editados como números monográficos dedicados a temas de interés para los psicólogos latinoamericanos (y también de otras latitudes) - convirtiéndose así en "órgano de expresión" y "vehículo de comunicación" de la psicología que se desarrolla en habla castellana (y portuguesa), incluyendo invariablemente para cada artículo publicado, un resumen en inglés que la conecta al circuito universal de la ciencia.

Desde la aparición del primer volumen, durante los primeros 25 años de la $R L P, 34$ autores peruanos han contribuido con igual número de artículos ${ }^{6}$, ya sea como autores únicos o en colaboración además, también, de suscribir artículos representando a instituciones extranjeras (es el caso de Eduardo Salas, cuya membresía está ligada a una institución norteame-

Vide Angelini, A. (1979). O papel da Sociedade Interamericana de Psicologia no desenvolvimiento de psicologia na America Latina, Interamerican Joumal of Psychology. 13(1-2); 5-25.

6. Hasta 1988 (dos décadas de vigencia de la publicación), la RLP había publicado 31 artículos ( $6.43 \%$ de 482 contribuciones acumuladas) correspondientes a 24 autores peruanos. Vide León, R. y Rivadeneyra, P. (1989). La psicología peruana a través de los veinte años de la Revista Latinoamericana de Psicología, Revista de Psicologia (PUCP). 7(2); 105-121 


\begin{tabular}{|c|c|c|c|c|c|c|}
\hline \multirow{2}{*}{$\frac{\text { Vol/Año }}{25-1993}$} & \multicolumn{3}{|c|}{ Números } & \multirow{2}{*}{$\begin{array}{c}\begin{array}{c}\text { Total de } \\
\text { ejemplares }\end{array} \\
3\end{array}$} & \multicolumn{2}{|r|}{$\begin{array}{c}\text { Números Especiales } \\
\text { (Monografías) }\end{array}$} \\
\hline & 1 & 2 & 3 & & $25(1)$ & PSIC. POLITICA \\
\hline $24 / 1992$ & $1 \mathrm{y}$ & 2 & 3 & 2 & $24(1-2$ & PSIC. Y SIDA \\
\hline $23 / 1991$ & 1 & 2 & 3 & 3 & $23(1)$ & PERSONALIDAD \\
\hline $22 / 1990$ & 1 & 2 & 3 & 3 & $22(1)$ & PROBLEMAS SOCIALES \\
\hline $21 / 1989$ & 1 & 2 & 3 & 3 & & \\
\hline $20 / 1988$ & 1 & 2 & 3 & 3 & $20(1)$ & PSIC. DE IA SALUD \\
\hline $19 / 1987$ & 1 & 2 & 3 & 3 & $19(3)$ & PERCEPCION Y PSICOFISICA \\
\hline $18 / 1986$ & 1 & 2 & 3 & 3 & $18(2)$ & PSIC. COMPARADA \\
\hline $17 / 1985$ & 1 & 2 & 3 & 3 & $17(2)$ & PSIC. EVOLUTIVA \\
\hline $16 / 1984$ & 1 & 2 & 3 & 3 & & \\
\hline $15 / 1983$ & $1 y$ & 2 & 3 & 2 & $15(1-2$ & BIORRETROALIMENTACION \\
\hline $14 / 1982$ & 1 & 2 & 3 & 3 & $14(3)$ & PSIC. GERONTOLOGICA \\
\hline $13 / 1981$ & 1 & 2 & 3 & 3 & $13(1)$ & PSIC. CLINICA \\
\hline $12 / 1980$ & 1 & 2 & 3 & 3 & $12(1)$ & PSIC. SOCIAL \\
\hline $11 / 1979$ & 1 & 2 & 3 & 3 & $11(2)$ & PSIC. EDUCACIONAL \\
\hline $10 / 1978$ & 1 & 2 & 3 & 3 & $10(1)$ & PSIC. INDUSTR. Y ORGANIZAC. \\
\hline $9 / 1977$ & 1 & 2 & 3 & 3 & $9(1)$ & $\begin{array}{l}\text { COMPORTAMIENTO SEXUAL } \\
\text { HUMANO }\end{array}$ \\
\hline $8 / 1976$ & 1 & 2 & 3 & 3 & $8(2)$ & PSIC. EXPRIM. LATINOAMERICANA \\
\hline $7 / 1975$ & 1 & 2 & 3 & 3 & $7(2)$ & APRENDIZAJE TEMPRANO \\
\hline $6 / 1974$ & 1 & 2 & 3 & 3 & & \\
\hline $5 / 1973$ & 1 & 2 & 3 & 3 & & \\
\hline $4 / 1972$ & 1 & 2 & 3 & 3 & & \\
\hline $3 / 1971$ & 1 & 2 & 3 & 3 & & \\
\hline $2 / 1970$ & 1 & 2 & 3 & 3 & $2(2)$ & TERAPIA DEL COMPORTAMIENTO \\
\hline $1 / 1969$ & 1 & $2 y$ & 3 & 2 & & \\
\hline $\mathrm{TOT}$ & & & & 72 & & Números Monográficos. \\
\hline
\end{tabular}

ricana, y de Luis Oblitas, miembro de la Universidad Nacional Autónoma de México) o hacerlo colaborando en un grupo "internacional" de autores (compuesto además de un peruano-Roberto Llanos-por mexicanos y un ecuatoriano). De otro lado, la $R L P$ ha dedicado al Perú el (hasta hoy) único número monográfico que da cuenta del estado y desarrollo de la psicología en un país latinoamericano ${ }^{7}$, al margen de haber dado cabida a múltiples presentaciones del pasado e historia de varios otros países del área.

7. Alarcón, R., ed. (1980). La Psicología en el Perú, Revista Latinoamericana de Psicología. 12(2); 199-393

116 
La historia de la $R L P$, cuando se escriba, reclamará ineludiblemente la presencia de Rubén Ardila, psicólogo colombiano nacido en 1942, quién es el que ideó, fundó, dirige y edita la publicación periódica más conocida en el área de la psicología en Latinoamérica ${ }^{8}$, éxito publicista que, como seńala su forjador, bien podría explicarse porque incuestionablemente llenó un vacío de comunicación entre los psicólogos de habla castellana de todo el mundo", además de permitir a los psicólogos latinoamericanos "informarse" no sólo de lo que sucede en el continente europeo, y la hoy muy influyente psicología norteamericana sino, también, de las no menos importantes aportaciones que hacen a la psicología los trabajos desarrollados en Latinoamérica ${ }^{10}$.

El significado de la RLP es enorme. Hoy es una fuente de lectura inexcusable para los psicólogos (y especialistas en ciencias afines); traduce, encauza y da forma tangible a lo más significativo de la producción bibliográfica de esta parte del mundo; posee un carácter general dentro de la psicología mostrando una gran amplitud temática y de colaboradores de todo el mundo, especialmente latinoamericanos, procurando desarrollar las líneas más actuales de la psicología universal y, además, con toda justicia -por la admiración que despiertan obras de largo aliento y por su enorme visibilidad en la región- tiene ya un lugar destacado en la historia de la psicología latinoamericana.

Marco Vargas López

8. Formalmente, desde 1977 la RLP es publicada y administrada por la Fundación para el Avance de la Psicología. Vide Ardila, R. (1978). Los 10 primeros años de la Revista Latinoamericana de Psicología, Revista Latinoamericana de Psicología. 10(3); 321-326

9. Ardila, R. (1986). La Revista Latinoamericana de Psicología y su papel en el desarrollo de la Psicología hispanoparlante, Revista Latinoamericana de Psicología. 18(3); 485-492

10. Quiñones, E.; Vera, J.; Sánchez, A. y Pedraja, M. (1991). La segunda década de la Revista Latinoamericana de Psicología (1979-1988); Una aproximación objetiva a la psicología en Latinoamérica, Revista latinoamericana de Psicologia. 23(2); 155-181 